\title{
Correction to: Deep venous drainage variant rate and degree may be higher in patients with perimesencephalic than in non-perimesencephalic angiogram-negative subarachnoid hemorrhage
}

\author{
Yuanjian Fang ${ }^{1} \cdot$ Anwen Shao $^{1} \cdot$ Xiaoyu Wang ${ }^{1} \cdot$ Jianan $\mathrm{Lu}^{1} \cdot$ Haijian $\mathrm{Wu}^{1} \cdot$ Reng Ren $^{1} \cdot \mathrm{Yi} \mathrm{Huang}^{1}$.

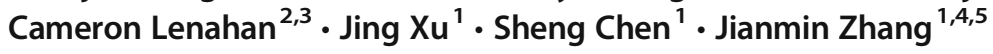 \\ Published online: 23 December 2020 \\ (C) European Society of Radiology 2020
}

\section{Correction to: European Radiology}

https://doi.org/10.1007/s00330-020-07242-5

The original version of this article, published on 11 September 2020, unfortunately contained mistakes. The following corrections have therefore been made in the original:

In the last paragraph of section "Variables," the third sentence should read: "Scores ranging from 4 to 5 for WFNS and 3-5 HH and 3-4 for mRS and SEBES were considered high for each respective scoring method."

In addition, the definition for WFNS was given incorrectly in Tables 1, 3, and 4 and the values of High WFNS were partly incorrect in Table 3. The corrected tables are given below. The original article has been corrected.

The online version of the original article can be found at https://doi.org/ $10.1007 / \mathrm{s} 00330-020-07242-5$

Sheng Chen

saintchan@zju.edu.cn

$\triangle$ Jianmin Zhang

zjm135@zju.edu.cn

1 Department of Neurosurgery, The Second Affiliated Hospital, School of Medicine, Zhejiang University, Hangzhou, Zhejiang Province, People's Republic of China

2 Center for Neuroscience Research, Loma Linda University School of Medicine, Loma Linda, CA, USA

3 Burrell College of Osteopathic Medicine, Las Cruces, NM, USA

4 Brain Research Institute, Zhejiang University, Hangzhou, Zhejiang, People's Republic of China

5 Collaborative Innovation Center for Brain Science, Zhejiang University, Hangzhou, Zhejiang, People's Republic of China 
Table 1 Summary of demographic, historical information, and outcome of angiogram-negative subarachnoid hemorrhage patients

\begin{tabular}{|c|c|c|c|c|c|}
\hline \multirow[t]{2}{*}{ Variables } & \multirow[t]{2}{*}{ Total $(n=273)$} & \multicolumn{2}{|l|}{ No. $(\%)$ of patients } & \multicolumn{2}{|l|}{$p$ value } \\
\hline & & $\begin{array}{l}\text { PAN-SAH } \\
(n=184,67.4 \%)\end{array}$ & $\begin{array}{l}\text { NPAN-SAH } \\
(n=89,32.6 \%)\end{array}$ & $\begin{array}{l}\text { Test for } \\
\text { correlation }^{\mathrm{a}}\end{array}$ & $\begin{array}{l}\text { Test for } \\
\text { difference }^{b}\end{array}$ \\
\hline Age & $56.2 \pm 11.1$ & $55.7 \pm 10.7$ & $57.4 \pm 11.8$ & 0.791 & 0.229 \\
\hline Female & $125(45.8)$ & $90(48.9)$ & $35(39.3)$ & 0.137 & 0.136 \\
\hline Hypertension & $97(35.5)$ & $62(33.7)$ & $35(39.3)$ & 0.364 & 0.362 \\
\hline Hypercholesterolemia & $42(15.4)$ & $29(15.8)$ & $13(14.6)$ & 0.805 & 0.804 \\
\hline Diabetes & $22(8.1)$ & $13(7.1)$ & $9(10.1)$ & 0.388 & 0.386 \\
\hline Tobacco use & $95(34.8)$ & $63(34.2)$ & $32(36.0)$ & 0.781 & 0.780 \\
\hline Alcohol use & $103(37.8)$ & $69(37.5)$ & $34(38.2)$ & 0.911 & 0.911 \\
\hline HН 3-5 & $34(12.5)$ & $8(4.3)$ & $26(29.2)$ & $<0.001$ & $<0.001$ \\
\hline WFNS 4-5 & $26(9.5)$ & $5(2.7)$ & $21(23.6)$ & $<0.001$ & $<0.001$ \\
\hline mFS 3-4 & $63(23.1)$ & $5(2.7)$ & $58(65.2)$ & $<0.001$ & $<0.001$ \\
\hline SEBES 3-4 & $17(6.2)$ & $2(1.1)$ & $15(16.9)$ & $<0.001$ & $<0.001$ \\
\hline IVH & $61(22.3)$ & $26(14.1)$ & $35(39.3)$ & $<0.001$ & $<0.001$ \\
\hline BVRs degree & & & & $<0.001$ & $<0.001$ \\
\hline 0 & $60(22.0)$ & $28(15.2)$ & $32(36.0)$ & & \\
\hline 1 & $50(18.3)$ & $34(18.5)$ & $16(18.0)$ & & \\
\hline 2 & $70(25.6)$ & $45(24.5)$ & $25(28.1)$ & & \\
\hline 3 & $44(16.1)$ & $35(19.0)$ & $9(10.1)$ & & \\
\hline 4 & $49(17.9)$ & $42(22.8)$ & $7(7.9)$ & & \\
\hline Positive repeated $\mathrm{DSA}^{\mathrm{c}}$ & $12(4.4)$ & $5(2.7)$ & $7(7.9)$ & 0.108 & 0.197 \\
\hline Hydrocephalus & $21(7.7)$ & $3(1.6)$ & $18(20.2)$ & $<0.001$ & $<0.001$ \\
\hline DCI & $36(13.2)$ & $9(4.9)$ & $27(30.3)$ & $<0.001$ & $<0.001$ \\
\hline Rebleeding & $6(2.2)$ & $0(0)$ & $6(6.7)$ & $<0.001$ & 0.001 \\
\hline Poor outcome $^{\mathrm{d}}$ (3 months) & $39(14.3)$ & $13(7.1)$ & $26(29.2)$ & $<0.001$ & 0.001 \\
\hline Poor outcome $^{\mathrm{d}}(1$ year $)$ & $23(8.4)$ & $4(2.2)$ & $19(21.4)$ & $<0.001$ & 0.001 \\
\hline
\end{tabular}

${ }^{a} p$ values testing for correlation result from the Spearman test of correlation

${ }^{\mathrm{b}} p$ values testing for difference between groups result from a Kruskal-Wallis test

${ }^{\mathrm{c}} 130$ AN-SAH patients (82 PAN-SAH and 48 NPAN-SAH) underwent repeated DSA

${ }^{\mathrm{d}}$ Poor outcome represents mRS 2-6 
Table 3 Association of BVR type with severity of subarachnoid hemorrhage and outcome

\begin{tabular}{|c|c|c|c|c|c|c|}
\hline \multirow[t]{2}{*}{ Variables } & \multicolumn{2}{|c|}{ No. $(\%)$ of patients } & \multirow[t]{2}{*}{$p$ value $^{\mathrm{a}}$} & \multicolumn{2}{|l|}{ No. $(\%)$ of patients } & \multirow[t]{2}{*}{$p$ value } \\
\hline & $\begin{array}{l}\text { Normal BVRs } \\
(n=60)\end{array}$ & $\begin{array}{l}\text { Abnormal BVRs } \\
(n=213)\end{array}$ & & $\begin{array}{l}\text { At least } 1 \text { primitive BVR } \\
(n=137)\end{array}$ & $\begin{array}{l}\text { No primitive BVR } \\
(n=136)\end{array}$ & \\
\hline \multicolumn{7}{|l|}{ SAH severity } \\
\hline High HH (3-5) & $14(23.3)$ & $20(9.4)$ & 0.004 & $9(6.6)$ & $25(18.4)$ & 0.003 \\
\hline High WFNS (4-5) & $13(21.7)$ & $13(6.1)$ & $<0.001$ & $3(2.2)$ & $134(98.5)$ & $<0.001$ \\
\hline High mFS (3-4) & $24(40.0)$ & $39(18.3)$ & $<0.001$ & $20(14.6)$ & $43(31.6)$ & 0.001 \\
\hline High SEBES (3-4) & $7(11.7)$ & $10(4.7)$ & 0.095 & $1(0.7)$ & $16(11.8)$ & $<0.001$ \\
\hline IVH & $17(28.3)$ & $44(20.7)$ & 0.207 & $20(14.6)$ & $41(30.1)$ & 0.002 \\
\hline NPAN-SAH & $32(53.3)$ & $57(26.8)$ & $<0.001$ & $31(22.6)$ & $58(42.6)$ & $<0.001$ \\
\hline \multicolumn{7}{|l|}{ Outcome } \\
\hline Positive repeated DSA ${ }^{\mathrm{b}}$ & $8(24.2)$ & $4(4.1)$ & 0.001 & $2(3.2)$ & $10(14.7)$ & 0.024 \\
\hline DCI & $14(23.3)$ & $22(10.3)$ & 0.009 & $12(8.8)$ & $24(17.6)$ & 0.03 \\
\hline Rebleeding & $5(8.3)$ & $1(0.5)$ & 0.002 & $0(0)$ & $6(4.4)$ & 0.038 \\
\hline Hydrocephalus & $5(8.3)$ & $16(7.5)$ & 0.833 & $6(4.4)$ & $15(11.1)$ & 0.039 \\
\hline High mRS (2-6) at 3 months & $15(25.0)$ & $24(11.3)$ & 0.007 & $13(9.5)$ & $26(19.1)$ & 0.023 \\
\hline High mRS (2-6) at 1 year & $13(21.7)$ & $10(4.7)$ & $<0.001$ & $3(2.2)$ & $20(14.7)$ & $<0.001$ \\
\hline
\end{tabular}

${ }^{a} p$ values were tested using chi-square analysis or Fisher's exact test

b 130 AN-SAH patients underwent repeated DSA (33 normal BVR, 97 abnormal BVRs, 62 at least 1 primitive BVR, 68 no primitive BVR)

Table 4 Multivariate analysis for predictors of positive findings on repeated DSA, rebleeding, DCI, and poor outcome at 1 year

\begin{tabular}{lrr}
\hline Variables & OR $(95 \%$ CI $)$ & $p$ value $^{\#}$ \\
\hline Predicting positive repeated DSA* & & 0.002 \\
Normal BVRs & $7.440(2.071-26.729)$ & \\
Predicting rebleeding & $30.772(4.565-207.437)$ & 0.001 \\
High SEBES (3-4) & $15.207(1.570-147.327)$ & 0.019 \\
Normal BVRs & & \\
Predicting DCI & $17.392(4.368-69.240)$ & 0.001 \\
High WFNS (4-5) & $4.477(1.739-11.528)$ & 0.002 \\
High mFS (3-4) & $3.318(1.352-8.140)$ & 0.009 \\
NPAN-SAH & & \\
Poor outcome at 1 year & $0.086(0.014-0.528)$ & 0.008 \\
Female & $10.108(2.560-39.916)$ & 0.001 \\
High WFNS (4-5) & $77.389(9.890-605.541)$ & 0.001 \\
High SEBES (3-4) & $5.728(1.625-20.195)$ & 0.007 \\
Normal BVRs & & \\
\hline
\end{tabular}

*130 AN-SAH patients underwent repeated DSA

\# Multivariate logistic regression analysis using backward selection

Publisher's note Springer Nature remains neutral with regard to jurisdictional claims in published maps and institutional affiliations. 